\title{
Kernos
}

Revue internationale et pluridisciplinaire de religion grecque antique

$13 \mid 2000$

Varia

\section{Jon D. MIKALSON, Religion in Hellenistic Athens}

\section{Vinciane Pirenne-Delforge}

\section{(2) OpenEdition}

\section{Journals}

Édition électronique

URL : http://journals.openedition.org/kernos/1322

DOI : 10.4000/kernos. 1322

ISSN : 2034-7871

Éditeur

Centre international d'étude de la religion grecque antique

Édition imprimée

Date de publication : 1 janvier 2000

ISSN : 0776-3824

\section{Référence électronique}

Vinciane Pirenne-Delforge, « Jon D. mIKaLson, Religion in Hellenistic Athens », Kernos [En ligne], 13।

2000, mis en ligne le 21 avril 2011, consulté le 24 septembre 2020. URL : http://

journals.openedition.org/kernos/1322 ; DOI : https://doi.org/10.4000/kernos.1322

Kernos 
Jon D. Mikalson, Religion in Hellenistic Atbens, Berkeley, Univ. of California Press, 1998, 1 vol. $16 \times 23,5 \mathrm{~cm}$, xIr +364 p. (Hellenistic Culture and Society, 29). ISBN : 0-520-21023-9.

La religion d'Athènes est un domaine de recherche fréquenté, mais ce sont surtout les périodes archaïque et classique qui retiennent généralement l'at tention. Cet ouvrage est donc bienvenu pour actualiser les développements consacrés par M.P. Nilsson à la période hellénistique dans sa Gescbichte der griechiscben Religion, vol. II (19612). L'A. distingue fermement période hellénistique et période romaine : l'ouvrage s'ouvre sur les réformes de Lycurgue après Chéronée et se referme sur le sac d'Athènes par Sylla (86). Il affirme le souci constant du temps et du lieu, pour éviter de créer le mirage d'une « religion hellénistique » qui n'a jamais été pratiquée nulle part par personne. La trame du propos est donc strictement chronologique et s'articule en huit chapitres, essentiellement fondés sur la documentation épigraphique athénienne.

L'action de Lycurgue après Chéronée (chap. 1) permet de faire l'état des lieux : la comparaison entre son discours contre Léocarès et ce que l'on sait de sa carrière fait apparaître la revitalisation d'Athènes sur le plan financier, militaire et religieux. Le souci de la continuité religieuse avec le passé apparaît clairement, mais l'accent est davantage porté sur les aspects salvateurs de certains cultes, comme celui d'Asclépios ou d'Amphiaraos. Avec l'arrivée de Démétrios de Phalère (chap. 2), homme de main de Cassandre, les Athéniens se voient forcés d'octroyer des honneurs divins à Alexandre. C'est sous son « règne » également que le système des chorégies est remplacé par le poste d'agonothète préposé à l'organisation des fêtes, que les dépenses funéraires sont limitées et que les gynéconomes sont chargés de limiter la participation à des activités rituelles privées. Démétrios fut formé à l'école d'Aristote et l'A. aborde brièvement la portée de l'influence de la philosophie sur la religion. Il n'exclut pas qu'une des sources de la divinisation du souverain ne soit à localiser dans les écoles philosophiques avec leurs résidents orientaux qui finissaient par « diviniser » leur fondateur. Ce chapitre se clôt sur une critique des vues de Ferguson touchant à un prétendu déclin religieux à la fin du $\mathrm{IV}^{\mathrm{e}}$ siècle.

En 307, Démétrios Poliorcète est accueilli en libérateur, et des honneurs lui sont octroyés ainsi qu'à son fils Antigone : statues, couronnes d'or, autel pour ces

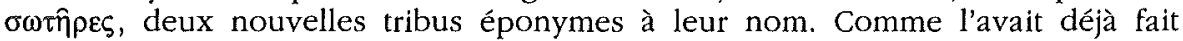
remarquer Chr. Habicht, jamais ils n'apparaissent comme dieux dans les inscriptions. Les éponymes sont, de plus, tous des héros, et l'on peut se demander si l'affirmation de l'A. selon laquelle les deux Macédoniens sont conçus comme des dieux n'est pas trop radicale, même si l'on peut s'accorder sur la nature "divine " des bienfaits désormais attendus de tels souverains. Il n'en reste pas moins que Démétrios a pris, semble-t-il, de tels honneurs au pied de la lettre, allant même jusqu'à s'installer sur l'Acropole. Après de tels excès, le calme revient pendant une vingtaine d'années à partir de la mort de Démétrios (chap. 4), et Athènes entreprend le redressement religieux de la cité en restaurant les Panathénées et en conférant à Zeus et à Athéna le nom de $\sigma \omega \tau \hat{p} \in \varepsilon_{\zeta}$, les vrais "sauveurs ". Le nom d'Archegetis que porte Athéna dans l'inscription SEG 28, 60, est nouvelle, d'après l'A., et vient se substituer au nom de Polias malmené par Démétrios. Cette Archegetis est honorée aux Chalkeia de $273 / 2$ et l'A. en conclut qu'elle est surtout liée à l'industrie et aux arts (p. 108-110). Toutefois, cette appellation d'Athéna apparaissait déjà dans le célèbre passage de la Lysistrata d'Aristophane qui décline les services religieux des jeunes Athéniennes (v. 642). On pourra arguer du fait qu'il s'agit de thêatre, d'une part, d'inscriptions officielles, d'autre part. Quoi qu'il en soit, l'appellation pourrait avoir été naturellement 
associée à Athéna depuis longtemps. L'A. s'intéresse ensuite à deux aspects de la «culture » athénienne du temps : la philosophie, d'une part, et la curiosité pour le passé de la cité avec les Atthidographes, d'autre part. Les premiers forment alors de petites sociétés souvent d'origine étrangère, qui ont peu d'influence sur la religion traditionnelle et respectent les traditions. Les seconds sont majoritairement des Athéniens partisans de la "démocratie " et cuvrent largement pour le respect des traditions religieuses de la cité.

Dans le chapitre 5, l'A. aborde la période entre la guerre chrémonidienne et l'année 229 qui voit le départ de la garnison macédonienne du Pirée. Il y étudie notamment la question de la prétendue "décadence " due au développement des associations religieuses privées au $\mathrm{III}^{\mathrm{e}}$ siècle (Ferguson). Un inventaire rigoureux des quelque 24 koina attestés par l'épigraphie montre qu'il s'agissait de petits groupes assez faibles et souvent d'une durée de vie limitée, qui n'étaient en tout cas pas en mesure d'affecter vraiment la religion traditionnelle. Par contre, les associations de soldats athéniens en garnison dans les forts de l'Attique ont développé une activité cultuelle importante en utilisant des cultes existants, mais aussi en créant des cultes, notamment à Rhamnonte. Quant aux honneurs rendus aux souverains, ils sont en net recul : c'est au simple titre de bénéficiaires des sacrifices, aux côtés du démos, qu'ils apparaissent comme destinataires d'hommages religieux. Vers 220 , un nouveau culte public voit le jour, celui du Démos associé aux Charites (chap. 6), dans le cadre duquel sera dédié, en 194/3, un magnifique autel pour Aphrodite Hegemonê tou dêmou et les Charites. L'A. propose l'intéres sante hypothèse que l'épiclèse d'Aphrodite serait le résultat d'une volonté d'associer les deux Charites ancestrales (Thallo, Auxo) et les deux Horai (Karpo, Hegemone) que mentionne Pausanias sous la forme d'une triade plus " canonique " de Charites (Thallo, Auxo, Karpo) et d'une Aphrodite Hegemonê. Cette période antérieure à la bataille de Pydna, est également marquée par les honneurs rendus à Ptolémée III Évergète en 224 et à Attale I de Pergame en 200, avec la création de deux nouvelles tribus éponymes, mais aussi par le saccage de l'Attique par Philippe V en 200 : cette invasion semble avoir sonné le glas de la vie religieuse si riche des dèmes attiques. Autre point important : la reconnaissance croissante des jeunes dans la religion civique, marquée à la fois par le rôle des éphèbes lors des fêtes et par la mise en évidence du service arrhéphorique dans les inscriptions.

Après la bataille de Pydna en 168, Athènes récupère Délos (chap. 7), chasse les habitants de l'île et en prend le contrôle. La description de la vie religieuse qu'y trouvent les Athéniens se fonde sur l'ouvrage de $\mathrm{Ph}$. Bruneau, de même que les modifications qu'ils y apportent, notamment la création de 10 prêtres prenant en charge des combinaisons de cultes. C'est à Délos qu'un réel intérêt des Athéniens pour certains cultes étrangers va se marquer, Sarapis, Atargatis, sans doute pour une part en raison de l'éloignement de leurs cultes traditionnels. Le dernier chapitre étudie l'«été indien » qui précède la mise à sac de la cité par Sylla, Les services éphébiques sont particulièrement révélateurs de la vie religieuse de la cité entre 168 et 86 : la religion est une des manières de maintenir les idéaux de liberté et de gloire militaire. De ce point de vue, la réapparition des Theseia dans la documentation en $165 / 4$ ou $161 / 0$ est révélatrice du sentiment d'un retour à la situation pré-macédonienne : la ville est réunifiée comme en un second synécisme. Les conclusions substantielles prennent une hauteur que les analyses de détail faisaient parfois perdre de vue et replacent utilement l'expérience athénienne dans un cadre plus large, fait de traditionnalisme et d'un individualisme qui relève moins d'un nouveau type d'expérience religieuse que d'une volonté de reconnaissance personnelle ou familiale d'un rôle religieux dans la cité. 
Dans la ligne de ses travaux antérieurs, l'A. étudie ce qu'il appelle la "religion populaire ", c'est-à-dire ce qui constitue l'activité religieuse du peuple. Il s'en tient dès lors surtout aux traces de cette activité que révèlent les inscriptions. De ce point de vue, ses analyses sont extrêmement fouillées et utiles : elles retouchent notamment le tableau traditionnel d'une période hellénistique plus ou moins «décadente» en regard de la splendeur classique, sous la pression de cultes étrangers et d'associations privées. La religion civique de la période considérée est avant tout caractérisée par la continuité, sciemment orchestrée, avec les périodes antérieures. La prospérité est conçue comme un bienfait lié au respect des traditions, et chaque crise sera suivie d'une volonté de restauration très clairement documentée par les inscriptions. Le recours systématique et presque exclusif à ce type de sources fait la force de cet ouvrage. Mais il en est peut-être aussi la faiblesse : le caractère formel et extrêmement codifié de tels documents ne nous permet pas, sans doute, d'atteindre toutes les dimensions de la Religion in bellenistic Atbens, mais ce qu'ils nous apportent est remarquablement analysé par ce livre.

Vinciane Pirenne-Delforge (Université de Liège)

Yulia Ustinova, The Supreme Gods of the Bosporan Kingdom. Celestial Aphrodite \& the Most High God, Leiden, Brill, 1999. 1 vol. $16 \times 24 \mathrm{~cm}, \mathrm{x}+$ 321 p., 28 pl, 2 cartes (Religions in the Graeco-Roman World, 135). ISBN : 90-04-11231-6.

Pendant les premiers siècles de notre ère, une «Aphrodite Ourania » et un " dieu suprême » ont dominé le panthéon du royaume du Bosphore, c'est-à-dire les régions du littoral nord de la Mer Noire où les Grecs s'étaient installés dès le $\mathrm{vrI}^{\mathrm{e}}$ siècle av. J.-C. Et pendant de longues années, de tels phénomènes religieux ont été essentiellement étudiés par des savants de l'ex-URSS dont les travaux, publiés en russe, restaient largement inconnus des chercheurs « occidentaux». Cette seule remarque montre déjà l'intérêt et l'importance du livre de Yulia Ustinova : non seulement elle offre une synthèse sur des questions qui n'avaient jamais été envisagées dans leur globalité jusqu'ici, mais cette synthèse est abondamment nourrie de la riche documentation archéologique et épigraphique recensée dans une bibliographie forcément mal maîtrisée par ceux qui ne pratiquent pas le russe. De plus, l'analyse de ces données religieuses doit prendre en compte les différentes composantes ethniques de la région (scythe, sindoméotienne, grecque, sarmate et, plus généralement encore, iranienne), ce qui confère à ce livre le statut de "cas d'école " pour l'étude des questions d'influences et de recouvrements religieux, bref tout ce que l'habitude conduit à regrouper sous le terme ambigu de "syncrétisme religieux ».

Après une brève introduction sur l'arrière-plan politique des siècles d'histoire envisagés, la première partie de l'ouvrage porte sur les déesses du Bosphore, en trois chapitres. Ils traitent respectivement des déesses grecques, des déesses locales et enfin de cette déesse toute-puissante que révèle la documentation des premiers siècles de notre ère. En comparaison des maigres attestations des cultes de déesses grecques comme, par exemple, Déméter, Artémis, Hécate ou Athéna, c'est Aphrodite qui a laissé le plus de traces dans les régions étudiées. Du $\mathrm{vi}^{\mathrm{e}}$ au $\mathrm{I}^{\mathrm{er}}$ siècle avant notre ère, le panthéon des colonies grecques du nord de la Mer Noire est dominé par celle que certaines inscriptions appellent Aphrodite Ourania protectrice d'Apatourum et Strabon, Aphrodite Apatouros. La déesse n'étant pas bien attestée dans les métropoles grecques, l'A. en déduit que l'influence locale a dû jouer dans le développement de cette figure, plus que dans le cas des 